\title{
Molecular characterization of Blastocystis subtypes in HIV-positive patients and evaluation of risk factors for colonization
}

\author{
Lucia Fontanelli Sulekova', Simona Gabrielli ${ }^{2,3^{*}}$ (D) Federica Furzi ${ }^{3}$, Giovanni Luigi Milardi ${ }^{3}$, Elisa Biliotti ${ }^{1}$, \\ Maurizio De Angelis ${ }^{1}$, Giancarlo laiani ${ }^{1}$, Caterina Fimiani ${ }^{3}$, Myriam Maiorano ${ }^{1}$, Simonetta Mattiucci ${ }^{2,3}$ and \\ Gloria Taliani ${ }^{1}$
}

\begin{abstract}
Background: Blastocystis is one of the most common intestinal protozoa in human faecal samples with uncertain impact on public health. Studies on the prevalence of Blastocystis in HIV-positive patients are limited and dated.

Methods: A cross-sectional study was carried out involving $156 \mathrm{HIV}$-positive patients to evaluate the prevalence of Blastocystis-subtypes by molecular amplification and sequencing the small subunit rRNA gene (SSU rDNA), to identify the risk factors for its transmission, to examine the relationship between the presence of the protist and gastrointestinal disorders. Furthermore, the evaluation of the faecal calprotectin by immunoassay from a sample of subjects was performed to evaluate the gut inflammation in Blastocystis-carriers.

Results: Blastocystis-subtypes ST1, ST2, ST3, ST4 were identified in 39 HIV-positive patients (25\%). No correlation was found between the presence of the protist and virological or epidemiological risk factors. Blastocystis was more frequently detected in homosexual subjects $(p=0.037)$ infected by other enteric protozoa $(p=0.0001)$ and with flatulence $(p=0.024)$. No significant differences in calprotectin level was found between Blastocystis-carriers and free ones.

Conclusions: Blastocystis is quite common in HIV-positive patients on ART showing in examined patients 25\% prevalence. Homosexual behaviour may represent a risk factor for its transmission, while CD4 count and viremia didn't correlate with the presence of the protist. The pathogenetic role of Blastocystis remains unclear and no gut inflammation status was detected in Blastocystis-carriers. The only symptom associated with Blastocystis was the flatulence, evidencing a link between the presence of the protist and the composition and stability of gut microbiota.
\end{abstract}

Keywords: Blastocystis, Subtype, PCR, HIV, Italy

\section{Background}

Blastocystis spp. is a common intestinal protist distributed worldwide infecting humans and animals, with a prevalence from $0.5-30 \%$ and $30-76 \%$ in industrialized and developing countries, respectively [1]. This difference can be explained by poor hygiene practices and consumption of contaminated food or water [2-5] since

\footnotetext{
* Correspondence: simona.gabrielli@uniroma1.it

${ }^{2}$ Clinical Diagnostic Parasitology laboratory, Umberto I Academic Hospital, 00185 Rome, Italy

${ }^{3}$ Department of Public Health and Infectious Diseases, Sapienza University of Rome, Piazzale Aldo Moro 5, 00185 Rome, Italy

Full list of author information is available at the end of the article
}

the faecal-oral route is considered to be the main mode of transmission of this protist [6]. A remarkable genetic diversity has been revealed among Blastocystis spp. isolates from humans and animals based on the comparison of the small-subunit rRNA gene (SSU rDNA) sequences [7]. Currently at least 26 subtypes of Blastocystis have been described among mammalian and avian isolates [8, 9], nine of them (ST-1 to ST-8 and ST-12) detected in human population and are potentially zoonotic [10]. Because several STs are shared between humans and animals it has been proposed that a proportion of human infections may result from zoonotic transmission.

(c) The Author(s). 2019 Open Access This article is distributed under the terms of the Creative Commons Attribution 4.0 International License (http://creativecommons.org/licenses/by/4.0/), which permits unrestricted use, distribution, and 
Indeed, a higher risk of Blastocystis infection was found in people with close animal contact, including zookeepers [11]. Despite several studies reported Blastocystis implicated in different intestinal diseases, potential pathogenetic factors have been described and its presence is frequently associated with symptoms in humans [12], its pathogenetic role is so far under debated and several variables, as well as the Blastocystis subtype and load, host's immune status and dysbiosis, could affect the occurrence of the disease $[13,14]$.

Although the protist was never considered as an opportunistic protozoon, it has also been frequently found in immunocompromised individuals presenting diarrhoea, with prevalence value ranging from 15 to $72.4 \%$ [15]. In addition, the sexual practices among men who have sex with men (MSM) have been reported to increase the transmission of Blastocystis (as for as other enteric organisms) [16]. Finally, recent data suggest that the protozoon is associated with certain gut microbiota profiles and health indices. Accordingly, a positive correlation between high bacterial richness and the presence of Blastocystis has been reported [17].

Notwithstanding it is commonly accepted that Blastocystis is a non-invasive organism, its vacuolar form is able to colonize the lamina propria, the submucosa and even the muscle layers leading to inflammation and active colitis in experimentally infected mice [18]. Currently, it is possible to perform a non-invasive evaluation of gut inflammation using biomarkers as the fecal calprotectin $(\mathrm{FC})$, which is considered a surrogate marker of intestinal inflammation with good diagnostic performance for separating organic and functional intestinal disorders [19]. The increase of FC is due to faecal excretion of neutrophils and macrophages migrants from the bloodstream into the intestinal lumen, which occurs during the intestinal inflammation. The value of FC as a laboratory marker has been shown in inflammatory bowel disease [20] but its significance in other gastrointestinal diseases remains unclear. Studies evaluating the correlation between intestinal inflammatory markers and parasitic infections are scant [21-24]. However, a positive correlation between the level of FC and severe infections due to intestinal parasites such as Giardia intestinalis and Schistosoma mansoni has been reported [21, 22]. Conversely, lower concentrations of FC have been reported in Blastocystis colonized individuals compared to non-colonized subjects [25].

This study aimed to evaluate the prevalence of Blastocystis subtypes in HIV-positive patients, to evaluate the potential the risk factors for its transmission and the relationship between the presence of the protozoon and gastrointestinal symptoms and, finally, to assess the level of FC in Blastocystis-colonized subjects.

\section{Methods}

\section{Study population}

A cross-sectional study was carried out from January 2016 to September 2017 involving HIV-positive patients followed at the Department of Translation and Precision Medicine, Umberto I Academic Hospital, Rome. A standardized questionnaire face-to-face interview was designed including demographic data (gender, age, profession, country of origin, recent travels) and information on clinical characteristics from each participating subject (CD4+ count and HIV-RNA load, therapy, laboratory results, comorbidity), potential risk factors for intestinal pathogens (exposure to domestic animals, diet, sexual behaviour) as well as detailed gastrointestinal symptoms (diarrhoea, abdominal pain, nausea, anorexia, weight loss, weakness, flatulence). According to World Health Organization definitions, diarrhoea was defined as the passage of three or more loose or liquid stools per day.

Written informed consent was obtained from every participant. The study was approved with respect to the Helsinki Declaration by the Ethical Committee of the Umberto I Academic Hospital (licence n. 4836).

\section{Laboratory analyses}

Blood and faecal samples were collected from each subject included in the study and submitted to the quantitative measurements of CD4+ T-lymphocytes and viral load [26], and to the microscopic observation of the wet smears stained with Lugol, directly or after Ridley concentration [27], respectively. Genomic DNA was then extracted from stool samples and submitted to PCR amplification using primers previously described [28], which target a fragment of about $600 \mathrm{bp}$ from the Blastocystis-SSU rRNA gene, following PCR protocol and conditions described in Mattiucci et al., 2016 [13]. The sequences obtained were compared to those of Blastocystis spp. deposited in GenBank using the BLAST application (www.ncbi.nlm.nih.gov/BLAST). The STs were identified by determining the exact match or closest identity (99\%), according to the classification given by Stensvold et al., 2007 [29].

Furthermore, the evaluation of the FC from a sample of subjects was performed using a commercial immunoassay (Calprest, EUROSPITAL, Italy), following the manufacturer's instructions. Samples giving values above 50 $\mathrm{mg} / \mathrm{kg}$ were regarded as having a positive Calprest test, as reported by the manufacturer and by previous published studies [30].

\section{Phylogenetic analyses}

Bayesian inference (BI) and Neighbour-Joining (NJ) phylogenetic combined tree, based on the amplified sequences of the Blastocystis-SSU rDNA gene, was carried 
out by using MrBayes3.1 [31], software and PAUP4* [32] respectively; the analysis were performed using the $H K Y+G(G=0.134)$ as the best model selection for the data set, as implemented in jModeltest, with Akaike Information Criterion (AIC) [33]. For Bayesian analysis, four incrementally heated Markov Chains (using default heating values), were run for 1,000,000 generations, sampling the Markov Chains at intervals of 100 generations. For each corresponding subtype, a referring sequence by GenBank database was included in the tree. B. lapemi and B. pythoni (GenBank accession no. AY590115 and KU146575, respectively) were used as outgroups.

\section{Statistical analyses}

Continuous variables were summarized as mean \pm standard deviation or median \pm interquartile range (IQR) and categorical data as counts and percentages. Comparisons between groups were performed using $X^{2}$ test or Fisher's exact test for categorical variables, and t-test or MannWhitney test for continuous variables. Multiple logistic regression was used to identify the predictors of Blastocystis colonization. The significance level for all analyses was set at $p<0.05$. Data were analysed using IBM SPSS, version 21.0 (SPSS Inc., Chicago, USA).

\section{Results}

A total of 156 consecutive HIV-positive patients were enrolled in the study between January 2016 and September 2017. Demographic and clinical data of the study population are described in Table 1 . The majority of subjects were males $(75 \%)$, mean age was $47.05 \pm 12.38$ (range 22 to 71 years), 39 participants $(25 \%)$ were coming from non-EU countries and 50 ones (32\%) were MSM (men who have sex with men). Moreover $47.4 \%$

Table 1 Epidemiological, demographic, immunologic and virological characteristics of the enrolled patients $(N=156)$. A total of $N=23$ were naïve and $N=133$ on ART

\begin{tabular}{ll}
\hline Study population & $\mathrm{N}(\%)$ \\
\hline Gender, male & $117(75 \%)$ \\
Age, years (mean \pm SD) & $47.05 \pm 12.38$ \\
Foreign origin & $39(25 \%)$ \\
MSM & $50(32.0 \%)$ \\
Domestic animals & $74(47.4 \%)$ \\
Travels & $67(42.9 \%)$ \\
CD4 count, cells/ml, mean \pm SD & $655.04 \pm 381.56$ \\
HIV-RNA (<37 copies/ml) & $112(71.8 \%)$ \\
Naïve subjects & $23(14.74 \%)$ \\
2NRTI + NNRTI & $35(26.3 \%)$ \\
2NRTI + IP/r & $34(25.6 \%)$ \\
2NRTI + INI & $36(27.1 \%)$ \\
Dual therapy & $28(21 \%)$ \\
\hline
\end{tabular}

were owner of pets and $42.9 \%$ travelled outside Europe in the last 6 months.

Twenty-three patients (14.7\%) were naïve and 133 (85.3\%) were on ART treatment. Thirty-five subjects (26.3\%) were on ART based on non-nucleoside reversetranscriptase inhibitors (NNRTI), 34 (25.6\%) on protease inhibitors (PI/r), 36 (27.1\%) on integrase inhibitors (INI) and $28(21 \%)$ on dual therapy. Three of the enrolled patients were on prophylaxis treatment with sulfamethoxazol-trimethoprim. The mean CD4 cell count was $655.04 \pm 381.56$ cells $/ \mathrm{mm}^{3}$. Most of patients (62.2\%) showed a CD4 count higher than 500 cells $/ \mathrm{ml}$, 26.3\% patients had a CD4 count between 200 and 500 cells $/ \mathrm{ml}$ and only $11.5 \%$ patients showed a CD4 count lower than 200 cells $/ \mathrm{ml}$. The median HIV-RNA level of naive patients was 40,010 (7093-824,500) -copies $/ \mathrm{mL}$. Among patients on ART treatment, HIV-RNA was detectable $(>37 \mathrm{cp} / \mathrm{mL})$ in $15.8 \%$ of subjects $(21 / 133)$ with a median HIV-RNA level of 116 (67-991) cp/mL.

Microscopic examination revealed the presence of Blastocystis spp. in 34 patients (21.8\%) while PCR amplification detected the protist in 39 individuals (25\%) and the expected $600 \mathrm{bp}$ fragments of the SSU rRNA gene amplified were successfully sequenced. Sequences obtained showed a high identity (98-100\%) to homologous sequences of Blastocystis isolates previously reported in GenBank and, according to the consensus terminology [29] and based on the phylogenetic analyses carried out, enabled the clustering of our isolates into 4 distinct Blastocystis-subtypes (Fig. 1).

In detail, ST3 was the most common subtype found in $51.3 \%$ of the subjects, followed by ST1 (30.8\%), ST4 (10.2\%) and ST2 (7.7\%). As expected, ST4 was identified only in European participants. In $30.7 \%$ of the subjects, Blastocystis was found in coinfection with other enteric protozoa as Entamoeba coli (15\%), Endolimax nana (8\%), Giardia intestinalis (6.1\%) and Iodamoeba butschlii (1.6\%). The detection of such intestinal parasites was more frequent in Blastocystis-carriers compared to Blastocystis-free ones $(p<0.0001)$ and in MSM compared to heterosexual subjects $(p<0.0001)$.

Neither demographic characteristics such as gender, age and nationality nor other epidemiological risk factors such as travel history or, presence of domestic animals differed significantly between the Blastocystis positive and Blastocystis negative patients (Table 2). In addition, no significant differences were found between the two groups regarding CD4+ T-cell counts, HIV-RNA undetectability and type of ART regiment. Blastocystis positive subjects reported more frequently homosexual behavior practices compared to negative ones $(48.71 \%$ vs $26.49 \%, p=0.037)$. After adjusting for age, the relationship between homosexual behavior practices and the presence of Blastocystis remained significant $(p=0.01)$. 


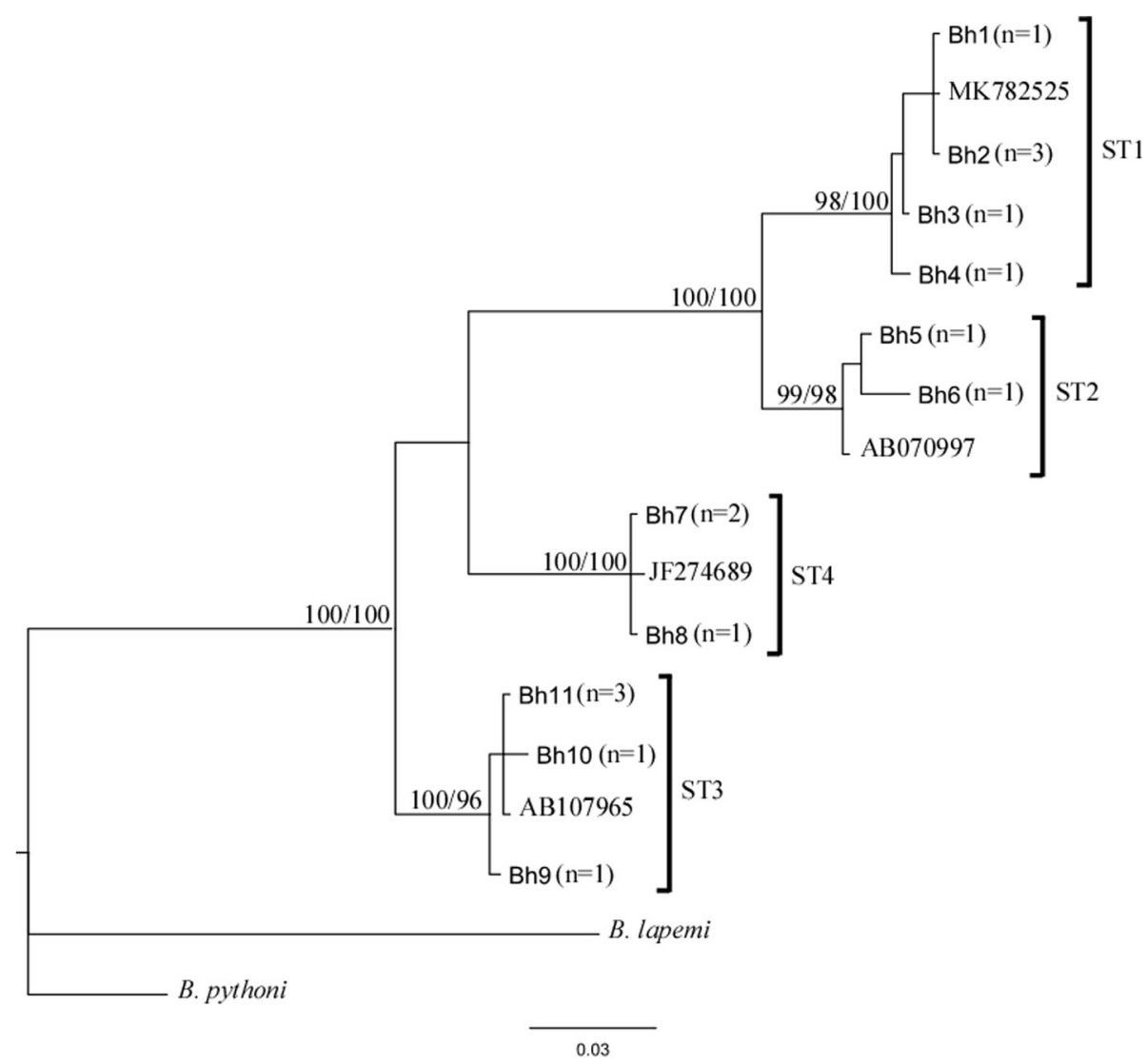

Fig. 1 Bayesian inference (BI) and Neighbour-Joining (NJ) phylogenetic combined tree showing the relationships among Blastocystis-SSU rRNA gene sequences from this study (Bh) and published Blastocystis-subtypes ST1, ST2, ST3, ST4 (GenBank accession numbers indicated in the tree), carried out by using MrBayes3.1 and PAUP4 respectively, as above described. The number of sequences from each isolate (Bh) was reported in the brackets. Bootstrap values and posterior probabilities, respectively, are reported at the nodes; B. lapemi and B. pythoni (accession no. AY590115 and KU146575, respectively) were used as outgroups

Most of the Blastocystis positive subjects were symptomatic (53.8\%), while $33.6 \%$ Blastocystis-free ones referred gastrointestinal disorders $(p=0.029)$. Among the gastrointestinal symptoms analyzed, flatulence was more frequently observed in Blastocystis carriers (30.7\%) compared to Blastocystis negative ones $(14.5 \%)(p=0.024)$ (Table 3).

To determine whether Blastocystis colonization was associated with intestinal inflammation, we measured FC in a subset of samples divided in Blastocystis-carrier (mono-infected) $(N=23)$ and Blastocystis-free $(N=36)$ subjects with comparable demographic, immunological and virological characteristics. From the all 59 fecal samples (Blastocystis-carrier and Blastocystis-free), $83 \%$ had a value in the standard range (lower than $50 \mathrm{mg} / \mathrm{kg}$ ) and $11(18.6 \%)$ had an increased value (median 115.7; 95\% CI $=71.82-303.78$ ). However, no significant differences in calprotectin values was found between Blastocystis-carriers (median 15, 95\% CI = 18.93-148.53) and non-carriers (median 13.35, 13.9331.74) $(p=0.31)$.

\section{Discussion}

Studies on the prevalence of Blastocystis spp. in HIVpositive subjects are relatively scant. In Italy the protist has so far attracted little attention, where few epidemiological surveys have been published, demonstrating a prevalence rate of about $7 \%$ and the occurrence of seven STs (ST1, ST2, ST3, ST4, ST6, ST7, ST8) in HIVnegative people [13, 34].

The only study conducted on Italian HIV-positive patients date back to 1999, through the microscopic method, showed a Blastocystis prevalence of 10.3\% [35]. The current study is, to our knowledge, the first to assess the prevalence of Blastocystis in HIV-positive patients through molecular methods in Europe and reported a prevalence of about $25 \%$, in line with that reported in previous studies conducted in Mexico (30\%) [36], Malaysia (19.8\%) [15] and Iran (19\%) [37]. The molecular characterization of Blastocystis positive isolates evidenced the circulation of 4 subtypes (ST1, ST2, ST3, ST4) already described in Italy, as previously mentioned $[13,38]$, being ST3 the most common one with a 
Table 2 Comparison of epidemiological, demographic, immunologic and virological characteristics between Blastocystis- carrier $(N=$ 39) and Blastocystis-free $(N=117)$ patients. Significant results were marked with *

\begin{tabular}{|c|c|c|c|}
\hline & Blastocystis-carriers N (\%) & Blastocystis-free N (\%) & $p$-value \\
\hline Gender, male & $33(82.0 \%)$ & $84(72.6 \%)$ & 0.289 \\
\hline Age, years (mean $\pm S D$ ) & $44.71 \pm 11.33$ & $47.05 \pm 12.37$ & 0.06 \\
\hline Foreign origin & $15(38.46 \%)$ & $25(21.36 \%)$ & 0.138 \\
\hline MSM & $19(48.71 \%)$ & 31 (26.49\%) & $0.037^{*}$ \\
\hline Domestic animals & $18(46.15 \%)$ & $56(47.86 \%)$ & 0.721 \\
\hline Travels & $20(51.28 \%)$ & $47(40.17 \%)$ & 0.296 \\
\hline CD4 count, cells/ml, mean \pm SD & $637.43 \pm 263.32$ & $660.90 \pm 414.35$ & 0.823 \\
\hline CD4 count $>500$ cells/ml & $28(71.79 \%)$ & 69 (58.97\%) & 0.484 \\
\hline CD4 count 200-500 cells/ml & $8(20.51 \%)$ & $33(28.20 \%)$ & \\
\hline CD4 count $<200$ cells/ml & $3(7.69 \%)$ & $15(12.82 \%)$ & \\
\hline HIV-RNA (<37 copies/ml) & $32(82.05 \%)$ & $80(68.37 \%)$ & 0.257 \\
\hline Naïve subjects & $5(12.82 \%)$ & $18(15.38 \%)$ & 0.765 \\
\hline 2NRTI + NNRTI & $9(23.07 \%)$ & $26(22.22 \%)$ & \\
\hline $2 \mathrm{NRTI}+\mathrm{IP} / \mathrm{r}$ & $7(17.94 \%)$ & $27(23.07 \%)$ & \\
\hline $2 \mathrm{NRTI}+\mathrm{INI}$ & $12(30.76 \%)$ & $24(20.51 \%)$ & \\
\hline Dual therapy & $6(15.38 \%)$ & $22(18.80 \%)$ & \\
\hline
\end{tabular}

prevalence of $51 \%$. In our cohort of HIV-positive patients, the presence of Blastocystis resulted no correlated to viro-immunological factors $(p>0.05)$ confirming therefore the non-opportunistic behavior of the protist. No demographic characteristic or epidemiological drivers (presence of domestic animals, dietary habits, travels abroad) were found to be associated with Blastocystis colonization. A higher prevalence of Blastocystis was reported in MSM compared to heterosexuals as well as of the other intestinal parasites, underlying the faecaloral contact as the main route of transmission in this group of subjects and the sexual practice and lifestyle linked to the presence of the protist more than the immunological status [16].

Despite the unresolved controversy over its pathogenicity, Blastocystis was also been frequently found in immunocompromised individuals presenting diarrhea [15]. In our study, among gastrointestinal symptoms, a positive association was found only for the flatulence. This socially disabling symptom is determined by two main factors: the diet, particularly the quantity of fermentable residues, and the composition and metabolic activity of colonic microbiota [39]. Since we evidenced any differences in the diet between Blastocystis-carriers and Blastocystis-free subjects, we hypothesized that this symptom was linked to the presence of the protist and the gut microbiota composition. Several Authors evaluated the intestinal microbiome in HIV-positive subjects, with somewhat inconsistent or controversial results [40]. This may be because of small sample sizes, lack of appropriate controls or regional differences in dietary and environmental factors. Regardless, many authors evidenced a change in the Bacteroides:Prevotella ratio, which they suggested to be linked to the antiretroviral therapy $[41,42]$ or to the sexual practice and lifestyle [43]. Similarly, several studies reported Blastocystis colonization associated with higher bacterial richness and Prevotella enterotype [44]. Therefore, investigations on the gut microbiota from HIV-positive subjects Blastocystis-carriers are needed to complete and to better understand the preliminary results obtained in this study.

Table 3 Gastrointestinal symptoms observed in Blastocystis- carrier $(N=39)$ and Blastocystis-free $(N=117)$ patients. Significant results were marked with *

\begin{tabular}{llll}
\hline Symptoms & Blastocystis-carriers N (\%) & Blastocystis-free N (\%) & $p$-value \\
\hline Abdominal pain & $7(17.94 \%)$ & $26(22.22 \%)$ & 0.836 \\
Diarrhoea & $10(25.64 \%)$ & $20(17.09 \%)$ & 0.283 \\
Flatulence & $12(30.76 \%)$ & $17(14.52 \%)$ & $0.024^{*}$ \\
Nausea & $3(7.69 \%)$ & $6(5.12 \%)$ & 0.887 \\
Poor appetite & $5(12.82 \%)$ & $10(8.54 \%)$ & 0.180 \\
Weight loss & $3(7.69 \%)$ & $10(8.54 \%)$ & 0.658 \\
\hline
\end{tabular}


Concerning FC, we found the median of both Blastocystis-carrier and free subjects within the normal range, supporting the non-pathogenic role of Blastocystis in inducing intestinal inflammation [25].

\section{Conclusions}

Despite some critical points of this study concerning the lack of data about the faecal microbiota from enrolled subject, to our knowledge this is the first large survey to molecular characterize Blastocystis subtypes in HIVpositive patients in Europe. Our results suggest that Blastocystis is quite common in such patients (25\%) and homosexual behavior resulted a risk factor for its transmission and confirm the presence of the protist not associated with pathological gut inflammation. The role of Blastocystis as intestinal pathogen remains unclear as the only symptom associated with its presence was flatulence. This result is intriguing since flatulence could be linked to the gut microbial communities, therefore the impact of the protist on the faecal microbiota and its possible role to maintain the to gut homeostasis could be interesting prospects for further studies.

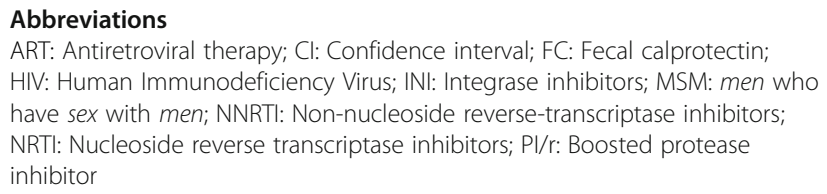

ART: Antiretroviral therapy; Cl: Confidence interval; FC: Fecal calprotectin; HIV: Human Immunodeficiency Virus; INI: Integrase inhibitors; MSM: men who have sex with men; NNRTI: Non-nucleoside reverse-transcriptase inhibitors; NRTI: Nucleoside reverse transcriptase inhibitors; PI/r: Boosted protease inhibitor

\section{Acknowledgements}

Authors are grateful to the Eurospital S.p.A. for providing the Calprest immunoassay.

\section{Authors' contributions}

LFS and SG designed the study with input from SM and GT. LFS, SG and FF conducted the data analysis, interpretation, and manuscript preparation. FF and GLM performed the laboratory analyses and molecular identification and contributed to the manuscript preparation. EB, MDA, GI, CF and MM enrolled the patients, performed the sample collection and contributed to the data analysis, data interpretation, and manuscript preparation. SG, SM and GT interpreted the results and revised the manuscript. All authors critically reviewed and approved the final version of this paper for publication.

\section{Funding}

This study was financed by Sapienza University in the frame of the "Progett di Ricerca Università Sapienza; years 2016, 2017 and 2018, grant numbers RM11615506189BE0, RM11715C81DDD2F3, RM11816432E08126". The content is solely the responsibility of the authors and the funder was not involved in the design of the study, data collection, analysis and interpretation of the data and in writing the manuscript.

\section{Availability of data and materials}

The dataset of this article will not be available publicly, to ensure the patient's privacy, but are available from the corresponding author on reasonable request.

\section{Ethics approval and consent to participate}

The study protocol was approved by the ethics committees of Umberto I Academic Hospital (licence n. 4836) in accordance with the ethical guidelines of the Declaration of Helsinki. Written informed consents were obtained from the patients after declaring the objectives of the present study.
Consent for publication

Not Applicable.

\section{Competing interests}

The authors declare that they have no competing interests.

\section{Author details}

${ }^{1}$ Department of Translation and Precision Medicine, Sapienza University of Rome, 00185 Rome, Italy. ${ }^{2}$ Clinical Diagnostic Parasitology laboratory, Umberto I Academic Hospital, 00185 Rome, Italy. ${ }^{3}$ Department of Public Health and Infectious Diseases, Sapienza University of Rome, Piazzale Aldo Moro 5, 00185 Rome, Italy.

Received: 25 July 2019 Accepted: 9 October 2019

Published online: 22 October 2019

\section{References}

1. Alfellani MA, Stensvold CR, Vidal-Lapiedra A, Onuoha ES, Fagbenro-Beyioku AF, Clark CG. Variable geographic distribution of Blastocystis subtypes and its potential implications. Acta Trop. 2013;126:11-8.

2. Leelayoova S, Siripattanapipong S, Thathaisong U, Naaglor T, Taamasri $P$, Piyaraj P, et al. Drinking water: a possible source of Blastocystis spp. subtype 1 infection in schoolchildren of a rural community in Central Thailand. Am J Trop Med Hyg. 2008;79(3):401-6.

3. Baldursson $S$, Karanis P. Waterborne transmission of protozoan parasites: review of worldwide outbreaks- an update 2004-2010. Water Res. 2011;45: 6603-14.

4. Lee LI, Chye T, Karmacharya BM, Govind SK. Blastocystis sp.: waterborne zoonotic organism, a possibility? Parasit Vectors. 2012;28:5-130.

5. Nagel R, Cuttell L, Stensvold CR, Mills PC, Bielefeldt-Ohmann H, Traub RJ. Blastocystis subtypes in symptomatic and asymptomatic family members and pets and response to therapy. Intern Med J. 2012:42(11):1187-95.

6. Yoshikawa H, Yoshida K, Nakajima A, Yamanari K, Iwatani S, Kimata I. Fecaloral transmission of the cyst form of Blastocystis hominis in rats. Parasitol Res. 2004;94(6):391-6.

7. Yoshikawa H, Wu Z, Nagano I, Takahashi Y. Molecular comparative studies among Blastocystis isolates obtained from humans and animals. J Parasitol. 2003;89(3):585-94.

8. Zhao GH, Hu XF, Liu TL, Hu RS, Yu ZQ, Yang WB, et al. Molecular characterization of Blastocystis sp. in captive wild animals in Qinling Mountains. Parasitol Res. 2017;116(8):2327-33.

9. Maloney JG, Lombard JE, Urie NJ, Shivley CB, Santin M. Zoonotic and genetically diverse subtypes of Blastocystis in US pre-weaned dairy heifer calves. Parasitol Res. 2019;118(2):575-82

10. Stensvold CR, Clark CG. Current status of Blastocystis: a personal view. Parasitol Int. 2016;65(6):763-71.

11. Parkar U, Traub RJ, Vitali S, Elliot A, Levecke B, Robertson I, et al. Molecular characterization of Blastocystis isolates from zoo animals and their animalkeepers. Vet Parasitol. 2010;169:8-17.

12. Wawrzyniak I, Poirier P, Viscogliosi E, Dionigia M, Texier C, Delbac F, et al. Blastocystis, an unrecognized parasite: an overview of pathogenesis and diagnosis. Ther Adv Infect Dis. 2013;1(5):167-78.

13. Mattiucci S, Crisafi B, Gabrielli S, Paoletti M, Cancrini G. Molecular epidemiology and genetic diversity of Blastocystis infection in humans in Italy. Epidemiol Infect. 2016;144:635-46.

14. Tito RY, Chaffron S, Caenepeel C, Lima-Mendez G, Wang J, Vieira-Silva S, et al. Population-level analysis of Blastocystis subtype prevalence and variation in the human gut microbiota. Gut. 2019:68(7):1180-9.

15. Tan TC, Ong SC, Suresh KG. Genetic variability of Blastocystis sp. isolates obtained from cancer and HIV/AIDS patients. Parasitol Res. 2009;105(5): 1283-6.

16. Stark D, Fotedar R, van Hal S, Beebe N, Marriott D, Ellis JT, et al. Prevalence of enteric protozoa in human immunodeficiency virus (HIV)-positive and HIV-negative men who have sex with men from Sydney, Australia. Am J Trop Med Hyg. 2007;76(3):549-52.

17. Andersen LO, Stensvold CR. Blastocystis in health and disease: are we moving from a clinical to a public health perspective? J Clin Microbiol. 2016; 54:524-8.

18. Elwakil HS, Hewedi IH. Pathogenic potential of Blastocystis hominis in laboratory mice. Parasitol Res. 2010;107(3):685-9. 
19. Gisbert JP, McNicholl AG. Questions and answers on the role of faecal calprotectin as a biological marker in inflammatory bowel disease. Dig Liver Dis. 2009;41(1):56-66

20. Lehmann FS, Burri $E$, Beglinger $C$. The role and utility of faecal markers in inflammatory bowel disease. Ther Adv Gastroenterol. 2015;8(1):23-36.

21. Bustinduy AL, Sousa-Figueiredo JC, Adriko M, Betson M, Fenwick A, Kabatereine $\mathrm{N}$, et al. Fecal occult blood and fecal Calprotectin as point-ofcare markers of intestinal morbidity in Ugandan children with Schistosoma mansoni infection. PLoS Negl Trop Dis. 2013;7:1-9.

22. Hanevik K, Hausken T, Morken MH, Strand EA, Mørch K, Coll P, et al Persisting symptoms and duodenal inflammation related to Giardia duodenalis infection. J Inf Secur. 2007;55(6):524-30.

23. Opintan JA, Newman MJ, Ayeh-Kumi PF, Affrim R, Gepi-Attee R, Sevilleja JE, et al. Pediatric diarrhea in southern Ghana: etiology and association with intestinal inflammation and malnutrition. Am J Trop Med Hyg. 2010;83(4): 936-43.

24. Kohli A, Bushen OY, Pinkerton RC, Houpt E, Newman RD, Sears CL, et al. Giardia duodenalis assemblage, clinical presentation and markers of intestinal inflammation in Brazilian children. Trans R Soc Trop Med Hyg. 2008;102(7):718-25.

25. Nieves-Ramírez ME, Partida-Rodríguez O, Laforest-Lapointe I, Reynolds LA, Brown EM, Valdez-Salazar A, et al. Asymptomatic Intestinal Colonization with Protist Blastocystis Is Strongly Associated with Distinct Microbiome Ecological Patterns. mSystems. 2018;3(3):1-18.

26. Falasca F, Di Carlo D, De Vito C, Bon I, d'Ettorre G, Fantauzzi A, et al. Evaluation of HIV-DNA and inflammatory markers in HIV-infected individuals with different viral load patterns. BMC Infect Dis. 2017;17(1):581.

27. Ridley DS, Hawgood BC. The value of formolether concentration of faecal cysts and ova. J Clin Pathol. 1956;9(1):74-6.

28. Scicluna SM, Tawari B, Clark CG. DNA barcoding of Blastocystis. Protist. 2006; 157(1):77-85.

29. Stensvold CR, Suresh GK, Tan KS, Thompson RC, Traub RJ, Viscogliosi E, et al. Terminology for Blastocystis subtypes - a consensus. Trends Parasitol. 2007; 23(3):93-6.

30. Mumolo MG, Bertani L, Ceccarelli L, Laino G, Di Fluri G, Albano E, et al. From bench to bedside: fecal calprotectin in inflammatory bowel diseases clinical setting. World J Gastroenterol. 2018;24(33):3681-94.

31. Ronquist F, Huelsenbeck JP. MrBayes 3: Bayesian phylogenetic inference undermixed models. Bioinformatics. 2003;19:1572-4.

32. Wilgenbusch JC, Swofford D. Inferring evolutionary trees with PAUP*. Curr Protoc Bioinformatics. 2003;6:6.4.

33. Posada D, Buckley TR. Model selection and model averaging in phylogenetics: advantages of akaike information criterion and bayesian approaches over likelihood ratio tests. Syst Biol. 2004;53:793-808.

34. Masucci L, Graffeo R, Bani S, Bugli F, Boccia S, Nicolotti N, et al. Intestinal parasites isolated in a large teaching hospital, Italy, 1 may 2006 to 31 December 2008. Euro Surveill. 2006;16:19891.

35. Brandonisio O, Maggi P, Panaro MA, Lisi S, Andriola A, Acquafredda A, et al. Intestinal protozoa in HIV-infected patients in Apulia, South Italy. Epidemiol Infect. 1999;123:457-62.

36. Sanchez-Aguillon F, Lopez-Escamilla E, Velez-Perez F, Martinez-Flores WA, Rodriguez-Zulueta P, Martinez-Ocaña J, et al. Parasitic infections in a Mexican HIV/AIDS cohort. J Infect Dev Ctries. 2013;7(10):763-6.

37. Piranshahi AR, Tavalla M, Khademvatan S. Genomic analysis of Blastocystis hominis isolates in patients with HIV-positive using locus SSU-rDNA. J Parasit Dis. 2018;42(1):28-33.

38. Meloni D, Sanciu G, Poirier P. Molecular subtyping of Blastocystis sp. isolates from symptomatic patients in Italy. Parasitol Res. 2011;109(3):613-9.

39. Manichanh C, Eck A, Varela E, Roca J, Clemente JC, González A, et al. Anal gas evacuation and colonic microbiota in patients with flatulence: effect of diet. Gut. 2014;63(3):401-8.

40. Williams B, Mirmonsef P, Boucher CA, Bushman F, Carrington-Lawrence S, Collman RG, et al. A summary of the first HIV microbiome workshop 2015. AIDS Res Hum Retrovir. 2016:32(10-11):935-41.

41. Vesterbacka J, Rivera J, Noyan K. Richer gut microbiota with distinct metabolic profile in HIV infected elite controllers. Sci Rep. 2017;7(1):6269.

42. Ling $Z$, Jin C, Xie T, Cheng Y, Li L, Wu N. Alterations in the fecal microbiota of patients with HIV-1 infection: an observational study in a Chinese population. Sci Rep. 2016;6:30673.
43. Noguera-Julian $M$, Rocafort $M$, Guillén $Y$, Rivera J, Casadellà $M$, Nowak $P$, et al. Gut microbiota linked to sexual preference and HIV infection. EBioMedicine. 2016:5:135-46.

44. Andersen LO, Bonde I, Nielsen HB, Stensvold CR. A retrospective metagenomics approach to studying Blastocystis. FEMS Microbiol Ecol. 2015; 91:1-9.

\section{Publisher's Note}

Springer Nature remains neutral with regard to jurisdictional claims in published maps and institutional affiliations.
Ready to submit your research? Choose BMC and benefit from:

- fast, convenient online submission

- thorough peer review by experienced researchers in your field

- rapid publication on acceptance

- support for research data, including large and complex data types

- gold Open Access which fosters wider collaboration and increased citations

- maximum visibility for your research: over $100 \mathrm{M}$ website views per year

At BMC, research is always in progress.

Learn more biomedcentral.com/submissions 